\title{
Adaptive Fuzzy / PD controller for Coupled Two Tank Liquid Levels system
}

\author{
Ahmed N Abdalla ${ }^{1,2}$, Thamir K Ibrahim ${ }^{1, *}$, Hai Tao ${ }^{3}$ \\ ${ }^{1}$ Faculty of Engineering Technology, University Malaysia Pahang, Gambang 26300, Malaysia. \\ ${ }^{2}$ CARRIFF, University Malaysia Pahang, Gambang 26300, Malaysia. \\ ${ }^{3}$ Faculty of computer system and software Engineering, University Malaysia Pahang, Gambang \\ 26300, Malaysia \\ ${ }^{4}$ Faculty of Mechanical Engineering, University Malaysia Pahang, Pekan 26600, Malaysia
}

\begin{abstract}
Liquid tank systems play important role in industrial application such as in food processing, beverage, dairy, filtration, effluent treatment, pharmaceutical industry, water purification system, industrial chemical processing and spray coating. A typical situation is one that requires fluid to be supplied to a tank at a constant rate which has Two-Input Two-Output (TITO). The accuracy and stability of TITO is one of the key factors of process which have cross coupling between process input and output. Unlike traditional neural network weight adaptation using gradient descent method, Fuzzy Adaptive PID control technique was utilized for adaptive tuning and fine tuning the controller's parameters. Design approach for controlling liquid levels of Coupled Tank TITO system by using Fuzzy Adaptive PID controllers. Tuning method for parameters of improved Fuzzy Adaptive, Particle Swamp Optimization and Neural network controllers ware also discussed. Experimental results demonstrate that the performances of proposed method perform well compared with convention Fuzzy and Tuned Fuzzy controllers; it was shown that Fuzzy Adaptive controller exhibited better performance in terms of transient response analysis.
\end{abstract}

\section{Introduction}

Process controls in industries is becoming more complicated and are eventually designed with intricate control systems in modern times. Controlling these processes requires an engineer who has comprehensive knowledge of the basic principles and the advanced techniques in process control design. Inadequate understanding of the concepts by the students who in turn get hired into operator, control engineer, process engineer and managerial positions may result in fatal loss of life and property. Today's control systems include more diagnostic sensors and automation, delivering increasing volumes of data. However, the gains accrued from closer process control and management can often be offset by losses due to time spent in dealing with the unexpected events in the process. Human operators/engineers continue to face the responsibility for making important and complex decisions, frequently within a very limited timeframe. There are approximately three million

* Corresponding author: thamirmathcad@yahoo.com 
regulatory controllers in continuous process industrial facilities (Karl Åström and Tore Hägglund, 2005), with typically between five hundred thousand regulatory controllers in each industry. Based on a survey of over eleven thousand controllers in the refining, chemical and pulp and paper industries, $97 \%$ of regulatory controllers utilize a PID feedback control algorithm.

Commercial application of the Proportional-Integral-Derivative (PID) controller for industrial activities has been ongoing for nearly seventy years. An example of the benefits of improving industrial control system performance can be found in the Industrial Information Resources report (Hiram Ponce et.al., 2015). The report reveals that major US process industries spend about thirty billion dollars annually on energy and over hundred billion dollars on facility maintenance. According to the report, even a $1 \%$ improvement in either energy efficiency or improved controller maintenance direction represents hundreds of millions of dollars in savings to process industries.

Industries process control of using Proportional Integral Derivative (PID) control continues remains a very challenging area of research. The popularity of this control algorithm is due to its simple structure as well as its ability to adequately control many types of processes. The main reason why PID controllers remain poorly tuned is the tedious and time consuming nature of traditional tuning methods. Instead, PID controllers are often tuned manually by trial and error. Therefore, automating the tuning process has large economic benefits since it saves valuable time and is a simple way of maintaining process performance. In the last decade a considerable amount of research in this area of process control has been documented in the literature and numerous commercial products have appeared on the market. However, the field is still maturing as researchers try to improve the performance of the automatic tuning methods and optimize the resulting PID controller parameters while at the same keeping the procedure simple.

In order to deal with this problem, the interactions should be the main design criteria when a controller is designed for a multivariable process system. The advanced control techniques have some difficulties in dealing with interactions among loops especially when band widths of those loops are limited. In some cases they are operated in supervisory mode (Houssemeddine et.al., 2015). Since PID controller is popular, design and tuning of PID controller is suggested. As a result most of PID controller operates in sub optimal manner. Most of industrial processes are often nonlinear systems (Okuyama, Y., 2008). Then design of classical linear controller is generally based on linearized model around a steady state point. Hence the PID controller cannot successfully control highly nonlinear plants (Akhtaruzzaman, M., Shafie, A.A., 2010).

Fuzzy PID controller (FPID) controllers have been successfully used in industrial processes and have often produced results superior to those of classical PID controller (Sinthipsomboon, K. et.al., 2011; Arulmozhiyal, R., Kandiban, R., 2012) including its simplified versions of fuzzy proportional derivative (FPD) and fuzzy proportional integral (FPI) controller (Malki, H.A, at.al., 1994;Sharma, S., Singh, B.,2010). This real benefit of FLC is due to the nonlinearity that exists in the fuzzy output, the heuristic nature associated with simplicity and effectiveness of both linear and nonlinear plants (Saab, S.S. 2004; Huaguang Zhang, et.al., 2011). The sub optimal PID performance can be improved while incorporating nonlinear FLC technique.

The control of liquid level in tanks and flow between tanks is a basic problem in the process industries. The process industries require liquids to be pumped, stored in tanks, then pump to another tank. Many times the liquids will be processed by chemical or mixing treatment in the tanks, but always the level of liquid in the tanks must be controlled, and the flow of the tank must be regulated. Level and flow control in tanks are at the heart of all chemical engineering systems. But chemical engineering system are also at the heart of our 
economies. Vital industries where liquid and flow control are essential include petro chemical industries, paper making industries and water treatment industries.

There are many alternative controller design theories that can be used to control the level of liquid on tanks. In addition, various controllers have been proposed in different areas (Feng, 2015), but due to the non-linearity in system components and alternators, these development feedback controllers could not efficiently control the liquid level and rather slow for the output responses. However, in the last decade, PSO, GA, and ANN optimization methods have been studied to tune the control parameters. It is been very simple for implementation (Ismail , 2012; Tan, 2011), but the dynamic response deteriorates when the complexity is increasing in the system.

In this respect, Fuzzy control is the most suitable system to get promising results in case of a properly choosing of the memberships and rules (Bevrani \& Daneshmand, 2012). Many strategies were used to determine the shapes and the limits of membership functions. Nevertheless, these methods are designed for a unique condition. So, any changes in tank system conditions should be led to change in membership shapes and limits. For this reason, it is desirable to introduce other type of controller such as artificial conventional fuzzy logic controller. To develop tuned Fuzzy intelligent PID controller that is based on the specification of the control signal for coupled two tank model. To validate the proposed method by performing simulations for couple tank water level control and the comparisons of control structures implemented using both PI and PID controllers.

\section{Related Work}

Process System Description: Several researchers have investigated the problem of controlling liquid flow of a single or multiple tanks (Khan MK, Spurgeon SK, 2006; Pan, et.al., 2005). A constrained predictive control algorithm based on feedback linearization applied to a coupled tanks apparatus is given (Kwok et.al., 2000) . Poulsen et.al., 2001, proposed a variable structure controller for second-order nonlinear system with saturation input and used the two-tank liquid level to illustrate their theoretical results.

Fig. 1 displays the graphical chart of the conjugated tank procedure believed in this find whereas Qi $=\{$ Qi1, Qi2 $\}$ are the aditus flow rate of tank one and two, Q12 is an orifice which the fluid flow can pass from tank one to tank two, $\mathrm{Q} o=\{\mathrm{Qo1}, \mathrm{Q} 02\}$ are the outlet flow rate of tank one and two and $h=\{h 1, h 2\}$ indicates the fluid water yield of tank one and two, separately. In this analog, with the purpose of manipulating the volume within tanks via the inlet fluid flowing from 2 pumps. The entries are $\mathrm{u}=\{\mathrm{u} 1(\mathrm{t}), \mathrm{u} 2(\mathrm{t})\}$ (voltage input (Vi) to pumps) and the exports are $\mathrm{h}=\{\mathrm{h} 1(\mathrm{t}), \mathrm{h} 2(\mathrm{t})\}$ fluid water yield in tank one and two separately [10].

Fuzzy PD/ PID/PI Controller: In a general FLC the control objective is to design a fuzzy controller using information based on some physical intuition event if the exact system dynamic is not known but the main problem is constructing the rule base for the controller. In a typical Mamdani type FLC, the rule base is obtained using the notion of classical PD, PI, or PID controller in Fuzzy control environment. The rule base is formed using the notion of Lyapunov Stability Theory for which V (x) represents a general single input single output nonlinear system. The fundamentals of fuzzy logic control, fuzzy sets, fuzzy rules and defuzzifications. 

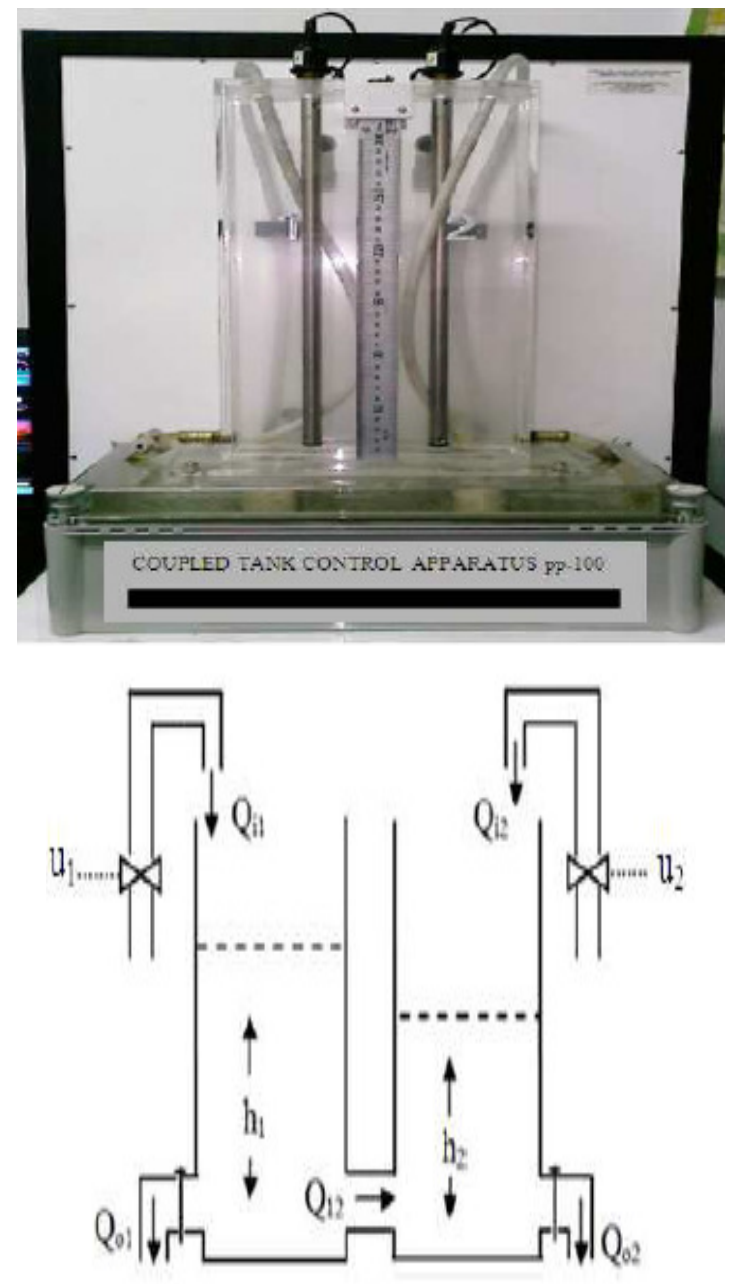

Fig. 1. Experimental setup for couple tank liquid-level system and Schematic of conjugated tank process.

There exists a continuously differentiable function $\mathrm{V}(\mathrm{x})$, known as Lyapunov function where $\mathrm{V}(\mathrm{x})$ is positive definite in the neighborhood of 0 and $\mathrm{V}^{-}(\mathrm{x})$ is negative definite in the neighborhood of 0. Fuzzy Lyapunov controller assumes prior knowledge of the system model. Under normal circumstances, only some partial knowledge about the system is known. According to the rule base, a fuzzy controller is obtained using general inference mechanism and defuzzifiction method. FLC structure that is with the rule base it will be possible to formulate the resulting conditions in the form of rules in one of two possible representations.

From figure 2, the controller design methods, the most common performance criteria are integrated absolute error (IAE), integrated of squared error (ISE) that can be evaluated analytically in the frequency domain (Yogendra; Rajesh Bhatt, 2013).

$$
I A E=\int_{0}^{t}|r(t)-y(t)| d t=\int_{0}^{t}|e(t)| d t
$$




$$
\begin{aligned}
& S E=\int_{0}^{t}(e(t))^{2} d t \\
& M S E=\frac{1}{t} \int_{0}^{t}(e(t))^{2} d t \\
& \text { ITAE }=\int_{0}^{t} t|e(t)| d t
\end{aligned}
$$

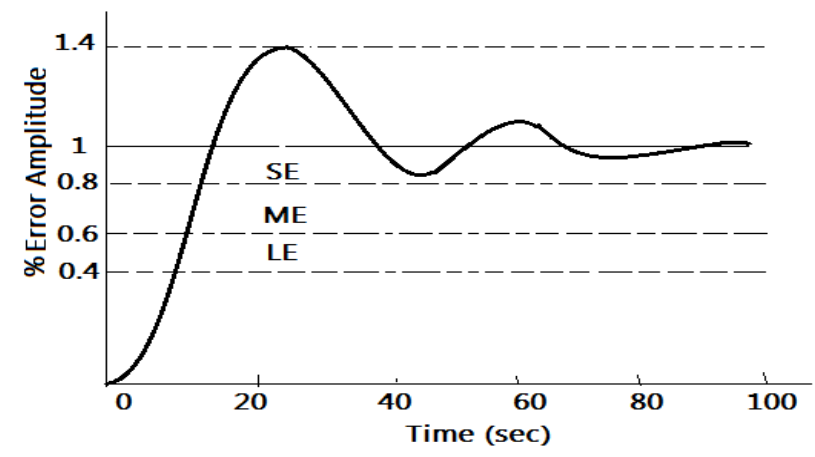

Fig. 2. Step response.

\section{Proposed Controller}

To overcome the backwards of Tuned of the fuzzy, the boundaries of the membership functions are adapting with input of the fuzzy controller by using PSO which select the suitable gains (Tuned fuzzy parameters). These gains represented by three parameters Gin1, Gin2 and Gout that shown in Figure 3 are defined the uncertain range by PSO algorithms.

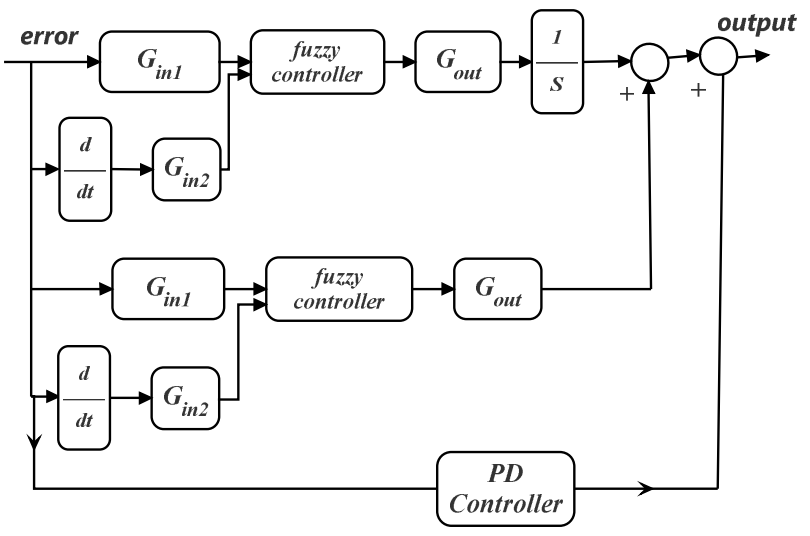

Fig. 3. Fuzzy PI / conventional PD controller.

Suppose that for a set of inflows Qi1 and Qi2, the fluid level in the tanks is at some steady state level H1 and H2. Consider a small variation in each inflow, q1 in Qi1 and q2 in Qi2. Let the resulting perturbation in level be h1 and h2 respectively. For figure 1, the liquid level of two tank coupled can be presented by the equation: 
For Tank 1

$$
A_{1} \frac{d\left(H_{1}+h_{1}\right)}{d t}=\left(Q_{i 1}+q_{1}\right)-\alpha_{1} \sqrt{H_{1}-h_{1}}-\alpha_{3} \sqrt{H_{1}-H_{2+} h_{1}-h_{2}}
$$

For Tank 2

$$
A_{2} \frac{d\left(H_{2}+h_{2}\right)}{d t}=\left(Q_{i 2}+q_{2}\right)-\alpha_{2} \sqrt{H_{2}-h_{2}}-\alpha_{3} \sqrt{H_{1}-H_{2+} h_{1}-h_{2}}
$$

Where

$H 1, H 2$ = height of fluid in tank 1 and tank 2 respectively

$A 1, A 2=$ cross sectional area of tank 1 and tank 2 respectively

$Q o 3=$ flow rate of fluid between tanks

Qi1, Qi2 = pump flow rate into tank 1 and tank 2 respectively

$Q o 1, Q o 2=$ flow rate of fluid out of tank 1 and tank 2 respectively

The procedure steps of the experimental work can be expressed as follows:

1. The storage tank was filled with 4 liters of water. The Tank 1 and Tank 2 ware filled with water by submersible water pump.

2. The computer system was operated which is supplied with all the hard-ware devices necessary for automatic control (analogue to digital converter (ADC), digital to analogue converter(DAC), and digital logic in and out(DLI/DLO)).

3. Set the water level at a certain input value (input variable).

4. The system was set automatically to record the steady-state values of water lavel (as a set point) after the steady-state conditions were reached.

5. a step change in the set point was carried .

6. The control systems were operated to eliminate the error values in water level (output variable) using Tuned Fuzzy Control level

7. The values of the errors in the level of water versus time were recorded and plotted by means of a plotter for different control strategies.

\section{Results and Discussions}

The conjugated tank system parameters are acquired as follows:

$\mathrm{H}=$ Height for tank $=18.5(\mathrm{~cm})$, Cross sectional area $=66.25\left(\mathrm{~cm}^{2}\right)$

$\mathrm{a}=$ Area of the coupling outlet $=0.1963\left(\mathrm{~cm}^{2}\right)$

$\beta 1=0.35903, \beta 2=0.345848, \beta x=0.38705, \mathrm{~g}=981 \mathrm{~cm} \mathrm{sec}^{12}$

The coupled tank fluid levels are needed to pursue pace replies in scope of 0-300 centimeter $(0-100 \%)$. The fluid level for tank one and two, both of them are noted in arrangement responses. The lowest and biggest benefits of the maneuvred retained variates are selected to $u$ min $=0$ volt, umax $=5 \mathrm{~V}$.

Error! Reference source not found. shows the liquid level responses of coupled tank system using Fuzzy and Tuned Fuzzy controllers for tank 1 and 2, respectively. It is noted that both controllers can track the step responses of $15 \mathrm{~cm}$. However, the Tuned Fuzzy shows a better performance in terms of time response specifications and integral square error as compared to the Fuzzy controller. For the time response performance of the liquid level in Tank 1, the Fuzzy produces settling time and rise time of $207 \mathrm{sec}$ and $15.7 \mathrm{sec}$, whereas the Tuned Fuzzy produces settling time and rise time of 28.3 and $22 \mathrm{sec}$. 


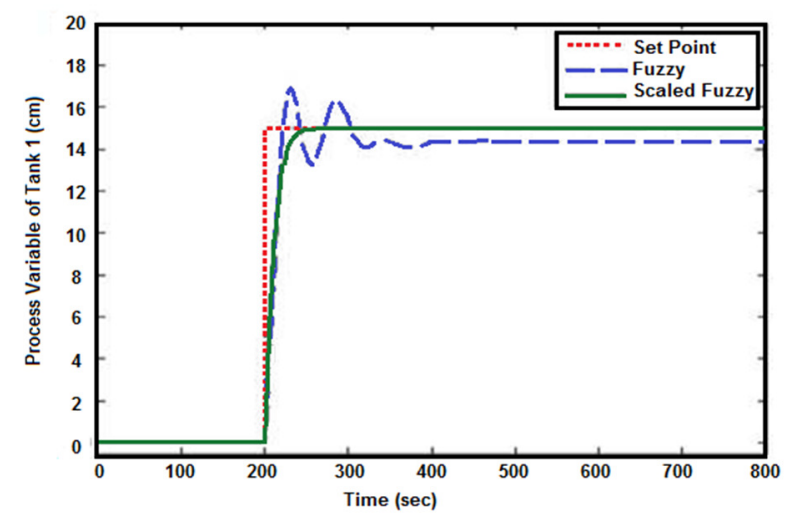

a)

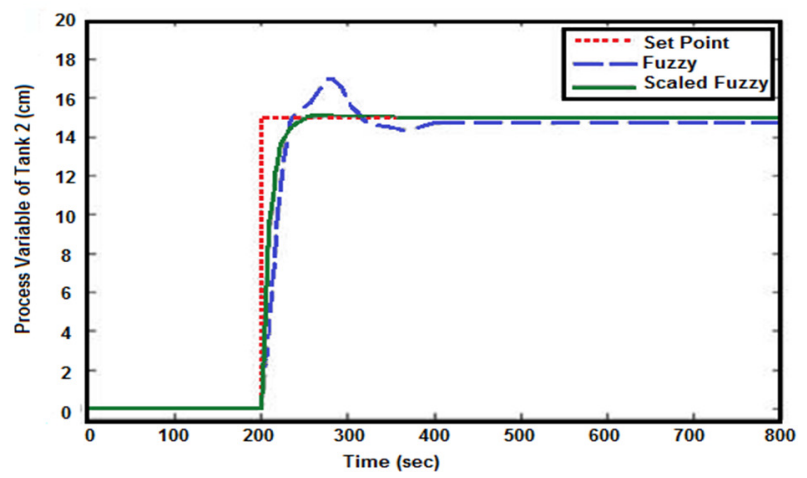

b)

Fig. 4. Simulated response of the liquid level in a) tank 1b) tank 2.

Error! Reference source not found. shows the liquid level responses in tank 1 and 2, respectively, with a step disturbance of $5 \mathrm{~cm}$ injected into the process variable of tank 1 during the steady-state response. Noted that the Fuzzy controller produced maximum percentage overshoots of $35 \%$ and $41 \%$ for liquid level in tank 1 and 2 whereas Tuned Fuzzy produces $27 \%$ and $18 \%$, respectively.

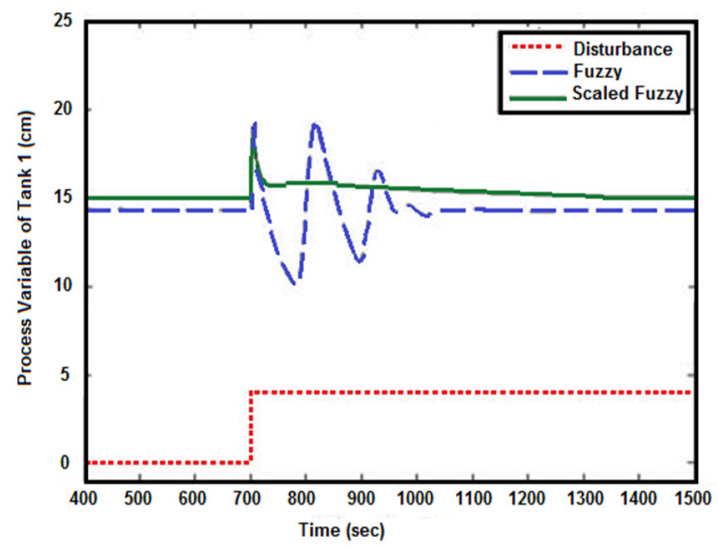

a) 


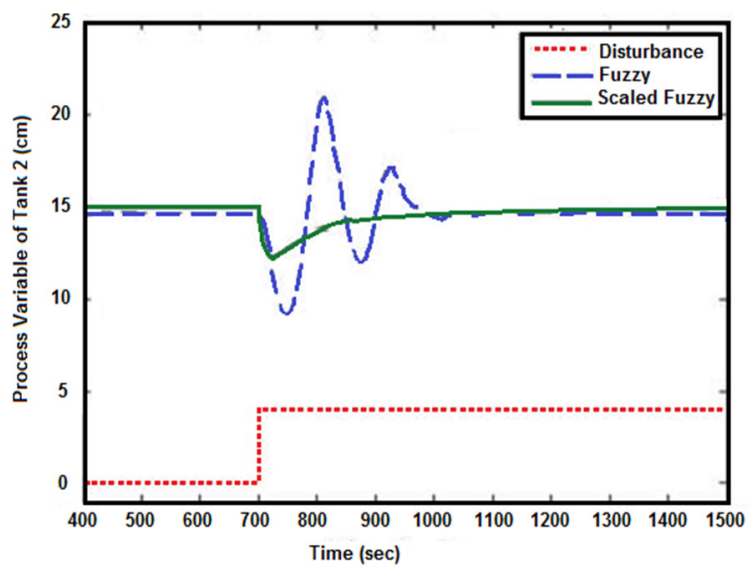

b)

Fig. 5. Simulated response of the liquid level in a) tank 1 with disturbance at tank 1 b) tank 2 with disturbance at tank 1 .

Furthermore, it could be seen that Tuned Fuzzy produce minimum oscillation as compared to Fuzzy in response to disturbance injection. It is proven that the Tuned Fuzzy controller results in faster settling time and minimum overshoot. Besides that Tuned Fuzzy also exhibits good robustness in minimizing the cross-coupling effect between two tanks.

In the meantime, Fuzzy produces settling time and rise of $209 \mathrm{sec}$ and $24 \mathrm{sec}$ whereas the Tuned Fuzzy produces settling time and rise time of $39.7 \mathrm{sec}$ and $22.2 \mathrm{sec}$ for the liquid level in tank 2. It shows that the Fuzzy results in a slower response as compared to Tuned Fuzzy. In term of integral square error for liquid level in tank 1, the Tuned Fuzzy results in twice less of ISE as compared to the Fuzzy with the value of $1.145 \times 105$ and $2.567 \times 105$ respectively. Similarly for liquid level in tank 2, the ISE of Tuned Fuzzy and Fuzzy were obtained as $1.209 \times 105$ and $2.567 \times 105$, respectively. The comparative assessment of both controllers is shown in Error! Reference source not found..

Table 1. Performance comparison of liquid level.

\begin{tabular}{|l|l|l|l|l|}
\hline \multirow{2}{*}{ Controller } & \multicolumn{2}{|c|}{ Settling time (sec) } & \multicolumn{2}{c|}{ Overshoot (\%) } \\
\cline { 2 - 5 } & Tank 1 & Tank 2 & \multicolumn{1}{c|}{ Tank 1 } & Tank 2 \\
\hline PID-F & 207.0 & 209.0 & 17.78 & 15.41 \\
\hline PID-SF & 38.3 & 39.7 & 0.00 & 0.067 \\
\hline
\end{tabular}

\begin{tabular}{|l|l|l|l|l|}
\hline & \multicolumn{2}{|c|}{ Rise time (sec) } & \multicolumn{2}{c|}{ MSE (x 105) } \\
\hline PID-F & 15.7 & 24.0 & 2.391 & 2.567 \\
\hline PID-SF & 22.0 & 22.2 & 1.145 & 1.209 \\
\hline
\end{tabular}

\section{Conclusions}

Control of liquid flow system is a routine requirement in many industrial processes. The control action of chemical and petroleum industries include maintaining the controlled variables. Scaled Fuzzy logic control can be applied for control of liquid flow and level in such processes. This technique is particularly attractive when the process is nonlinear. The objectives of this research were successfully achieved. These include the development of the optimization method by using PSO, Fuzzy and Tuned Fuzzy, simulation software design. Analysis of the table shows, the overshoot and comprehensive performance indicators 
adjustment time, Tuned Fuzzy algorithm is the best effect of real-time control, designers can use the knowledge learned in control theory and to design a variety of control algorithms, and validate the system.

Financial support for this research was provided by University Malaysia Pahang, RDU grant number 1403116. The authors would like to thank Universiti Malaysia Pahang for providing supports to conduct the project.

\section{References}

1. Akhtaruzzaman, M., Shafie, A.A. Modeling and control of a rotary inverted pendulum using various methods, comparative assessment and result analysis. International Conference on Mechatronics and Automation, 1342 - 1347 (2010).

2. Arulmozhiyal, R.; Kandiban, R. Design of Fuzzy PID controller for Brushless DC motor. International Conference on Computer Communication and Informatics, 1 - 7 (2012).

3. Feng Zhou, Hui Peng, Yemei Qin, Xiaoyong Zeng, Wenbiao Xie, Jun Wu, J. Process Contr. 34: 97-116 (2015).

4. Hiram Ponce, Pedro Ponce, Héctor Bastida, Arturo Molina, Expert Syst. Appl. 42, 88588867 (2015).

5. Houssemeddine Gouta, Salim Hadj Said, Faouzi M'sahli, The Journal of Franklin Institute, (2015).

6. Huaguang Zhang; Ruizhuo Song; Qinglai Wei; Tieyan Zhang, IEEE Trans. Neural Netw., 22(12), 1851 - 1862 (2011).

7. Karl Åström and Tore Hägglund, Advanced PID Control. ISA - The Instrumentation, Systems, and Automation Society (2005).

8. Khan, M. K., \& Spurgeon, S. K., Control Eng. Pract., 14(4), 375-386 (2006).

9. Kwok KE, Ping MC, Li P., J Process Contr., 10, 9-18. (2000)

10. Malki, H.A.; Huaidong Li; Guanrong Chen, IEEE T. Fuzzy Syst., 2(4), 245 - 254. (1994).

11. Okuyama, Y. American Control Conference on a grid pattern, 4746 - 4751 (2008).

12. Pan H, Wong H, Kapila V, de Queiroz MS., Control Eng. Pract., 13, 27-40 (2005).

13. Poulsen NK, Kouvaritakis B, Cannon M., IEE Proc. Control Theory Appl., 148,17-24 (2001).

14. Saab, S.S, IEEE Transactions on Automatic Control, 2261 - 2265, (2004).

15. Sharma, S.; Singh, B., International Conference on Power, Control and Embedded Systems, 1-8 (2010).

16. Sinthipsomboon, K.; Hunsacharoonroj, I.; Khedari, J.; Pongaen, W.; Pratumsuwan, P., 6th IEEE Conference on Industrial Electronics and Applications, 220 - 225 (2011).

17. Tan W., Nucl. Eng. Des, 241,1873-1880 (2011)

18. Yogendra Kumar Soni and Rajesh Bhatt, Int. J. Adv. Res Comput. Eng. Technol., 2, 2333-2335 (2013). 